\title{
Terahertz CMOS PA-less Dynamic Optimal Load OOK Modulator for \\ High-Speed Chip-to-Chip Communication
}

\author{
Jason Truman \\ University of California, Los Angeles
}

\begin{abstract}
A novel architecture of terahertz CMOS PA-less dynamic optimal load OOK modulator with low loss, high ON/OFF ratio and high data rate while no DC power consumption is proposed. The modulator is simulated in a $28-\mathrm{nm}$ CMOS technology and has $3 \mathrm{~dB}$ loss, $20 \mathrm{~dB}$ ON/OFF ratio and $20 \mathrm{Gbps}$ data rate with zero DC power by utilizing digitally controlled artificial dielectric (DiCAD). The proposed terahertz modulator helps make possible $20 \mathrm{Gbps}$ short-range wireless data transmission at terahertz regime.
\end{abstract}

\section{Introduction}

The demand for bandwidth in wireless short-range communication systems has doubled every 18 months over the last 25 years [3]. We can extrapolate wireless data rate of 20 40Gbps will be needed in 10 years from now. With more and more people using wireless networks, the demand for the ultra-fast wireless communications systems is increasing. To date, this demand has been accommodated with advanced modulation schemes and signal-processing technologies at microwave frequencies. However, without increasing the carrier frequencies for more spectral resources, it may be quite difficult to keep up with the needs of users. Although there are several alternative bands, recent advances in terahertz-wave (THz-wave) technologies have attracted attention due to the huge bandwidth of $\mathrm{THz}$ waves and its potential for use in wireless communications.

Electromagnetic waves in terahertz $(300 \mathrm{GHz}-3 \mathrm{THz})$ region of spectrum gives rise to a number of unique and important characteristics that offer benefits for short-range high data rate wireless communication. The available wide absolute bandwidth and high carrier frequency have a good potential to support ultra-broadband data transmission with a small aperture and system size. For example, at $300 \mathrm{GHz}$, the dimensions of on-chip antenna become comparable to a few pads, and antenna inefficiency is comparable to signal loss through the pads and the package. Another additional advantage in this frequency range is that the high directionality for a given compact physical aperture provides secure communication links. For such applications, high directivity techniques such as antenna array and/or beamforming become mandatory.

Traditionally, systems implemented in this spectrum range have been realized using discrete components based on less accessible technologies such as GaAs or InP HBTs and HEMTs, which unavoidably results in large form factor and high cost.[4] Recent circuit development based on deep-scaled CMOS continues to push the circuit and system 
applications into the terahertz frequency regime with broader bandwidth by monolithically integrating the terahertz front end and the baseband digital signal processor for smaller form factor and less power consumption.

\section{Review of current state-of-art millimeter wave/ terahertz OOK modulator}

For transceiver designs, an (On-Off-Key) OOK transceiver is a good candidate in terahertz band because the transmission and the detection are simple both in system level and a circuit level. In the low frequency range, in order to boost spectrum efficiency, complex modulation schemes, such as QPK, 16QAM, 64 QAM, are adopted. Basically, digital single processors and mixed signal circuit blocks, such as ADCs, DACs and PLLs are indispensable for transceivers using complex modulation schemes. Extremely huge power consumption is caused by using these digital signal processors and mixed-signal circuit blocks, which cannot be afforded by portable devices for more than 10Gbps digital and mixed-signal circuits. However, bandwidth resources are adequate in the terahertz band, thus there is no need to utilize power-consuming complex modulation schemes and an OOK modulation scheme is a good choice.

An OOK modulator, which is the most important block of an OOK transmitter, can be classified by three types: switching oscillator, switching amplifier, modulating switch. The switching oscillator mode makes an OOK-modulated signal by controlling the oscillator directly. [5] This can help to obtain high ON/OFF ratio because there is no generated output signal in the OFF state. However, it is difficult to support more than $10 \mathrm{Gbps}$ data due to the long start-up time of the oscillator. The switching amplifier mode is one in which an power amplifier can be switched in accordance with OOK data. [6] Basically, an amplifier based on cascade amplifier structure modulates OOK data by controlling the gate bias of the common-gate stage of the cascade amplifier. However, there is no efficient power gain available at $200 \mathrm{GHz} \sim 300 \mathrm{GHz}$, which is close to $\mathrm{f}_{\mathrm{T}}$ of $65 \mathrm{~nm}$ CMOS technology. The method of modulating switch is realized by using distributed shunt switches between the signal and the ground line of a transmission line. This method can successfully process around $8 \mathrm{Gbps}$ data [7].

\section{A. Gilbert based OOK modulator}

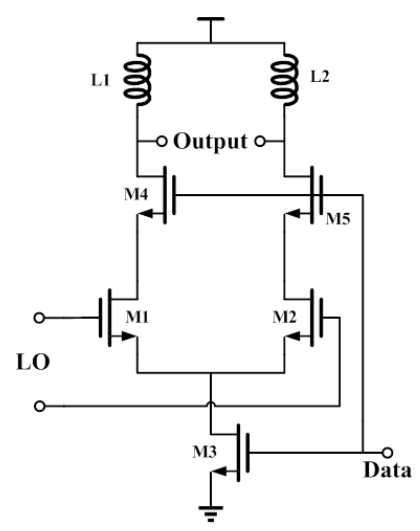


Figure 1. Gilbert based OOK modulator

Gilbert based OOK modulator is a type of switching power modulator as Fig.1 shows. In this topology, the input data directly modulates the $300 \mathrm{GHz}$ oscillator frequency before it is delivered to the antenna. Switches M3, M4, M5 completely block the input clock when they are tuned off, preventing potential signal leakage to the output. The main problem of this architecture is the loss because there is even no gain at $300 \mathrm{GHz}$. Simulation shows that the loss from LO input to the output is around $10 \mathrm{~dB}$, which is not acceptable for precious terahertz power. Another problem is that the modulation switches M4 and M5 are the cascode devices, suffering from body effect, which also limit the bandwidth of this modulator. Other drawbacks of this modulator include low on/off isolation (less than 20dB) and large DC power in the ON state.

\section{B. Distributed-switch OOK modulator}

Distributed Modulator

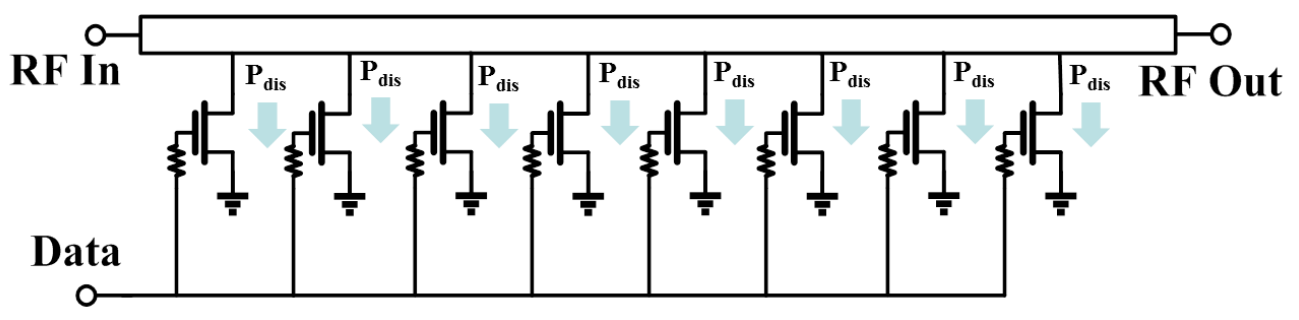

Figure 2. Distributed modulator

This distributed modulator is a type modulating switch modulator. In this architecture, when the switches are off the input signal is transferred to the output and the OOK modulator is in the ON state. On the other hand, when the switches are turned on, no input signal is transferred to the output and the OOK modulator is in the OFF state. The distributive feature of this modulator can run much faster compare with Gilbert based modulator. In other words, distributed modulator has larger bandwidth and faster settling time. However, low-quality parasitic capacitances in the shunt switches, which are located on a silicon substrate, degrade the transmission line characteristics because the switches do not only dissipate the incident signal, but they also reflect and leak it as shown in Fig. 2.

\section{Reduced-switch distributed modulator}

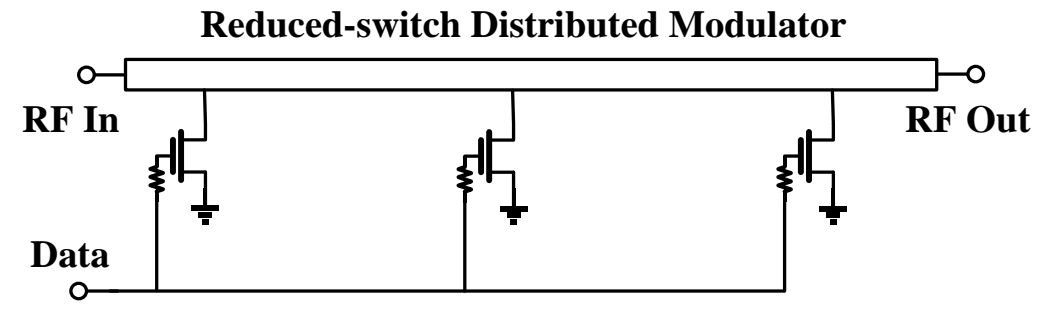

Figure 3. Reduced-switch distributed modulator

In order to solve the loss problem of distributed modulator, a reduced-switch architecture is put forward as Fig. 3 shows. It is noticed that the output power is much larger in the ON state because of less OFF switches dissipation, compared with distributed modulator. However, the isolation characteristics become degraded upon reducing the number of 
switches. To achieve high ON/OFF ratio, there is a tough trade-off for the length of transmission line and the locations of the switches. On one hand, in the ON state of the OOK modulator, there is a specific length for optimal load for conjugate maximum-power match. Furthermore, transmission line loss also should be taken into account because longer transmission line has larger loss. On the other hand, in the OFF state of the OOK modulator, there is another specific length for highest isolation impedance. The highest isolation length should usually be around one quarter wavelength. As a result, the optimal transmission line length for maximum power transfer in the ON state conflicts a lot with the optimal transmission line length for highest isolation in the OFF state.

\section{Description of problems}

It seems that reduced-switch distributed modulator is pretty promising to be a good terahertz OOK modulator, as long as one can overcome the tough trade-off of the length of transmission line and the locations of the switches. Here is the most significant problem of terahertz OOK modulator. It is really difficult to obtain the maximum power transfer and best isolation at the same time. Maximum power transfer is the most preferred feature because terahertz radiated power is so precious that no one can accept to waste it based on state-of-art low efficiency terahertz power generation. Simultaneously, a good isolation when the modulator is OFF, are equally critical because reasonable SNR ( $13 \mathrm{~dB}$ for $10^{-7}$ $\mathrm{BER}$ ) is needed for demodulated high data rate stream, which is achieved by reasonably high $\mathrm{ON} / \mathrm{OFF}$ ratio.

In the proposed novel terahertz OOK modulator, the tough trade-off mentioned above is possible to be overcome by utilizing digital configurable differential transmission line, called digitally controlled artificial dielectric (DiCAD). With the help of DiCAD, the effective length of transmission line is dynamically changed according to data. The detailed design of this novel modulator will be covered in the next section.

\section{Proposed dynamic optimal load OOK modulator}

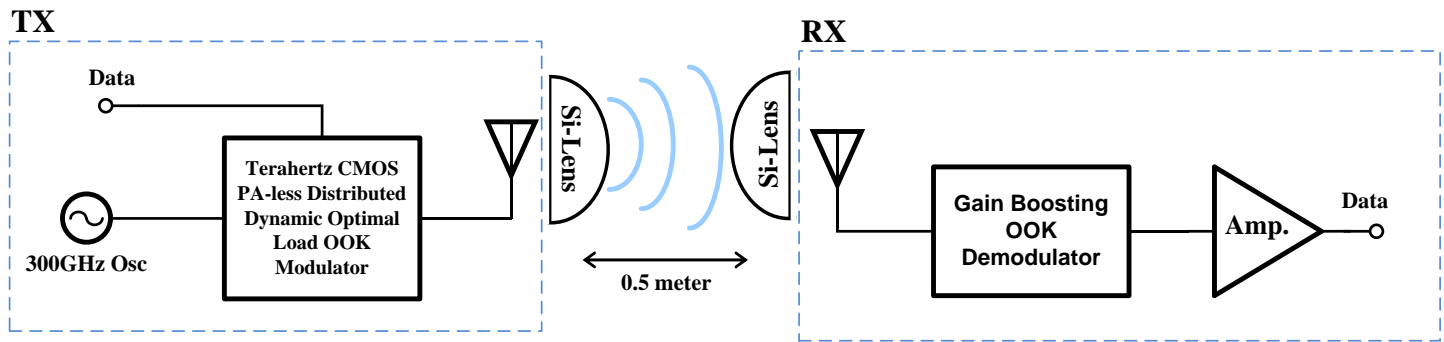

Figure 4. Proposed Transceiver Architecture

A self-heterodyne structure based on OOK modulation is shown in Fig. 4. The transmitter should not reject the carrier frequency, e.g., $300 \mathrm{GHz}$ signal in a transmitted single, because it is reused for a local signal in a receiver block. The receiver front end is a square law detector, gain boosting OOK demodulator (nonlinear device), then mixes the received 
terahertz signal with the received carrier and recovers an OOK data signal at the output. Also antennas or arrays and Si-Lens are proposed to use to compensate high path loss at $300 \mathrm{GHz}$. The dimensions of on-chip antenna become comparable to a few pads, and antenna inefficiency is comparable to signal loss through the pads and the package. Making a fully integrated solution is the preferred choice.

In the proposed novel terahertz modulator - terahertz CMOS PA-less distributed dynamic optimal load OOK modulator, the digitally controlled artificial dielectric (DiCAD) will play an important role in it because DiCAD is capable to show different effective lengths according to different digital control. The simplified schematic shows in Fig. 5. That means the tough trade-off of length of transmission line can be overcome because the electrical length of DiCAD could be data-dependent. In the ON state, DiCAD digital control bit is set to optimal impedance for highest power transfer. In the OFF state, DiCAD digital control bit is set to optimal impedance for highest isolation. Furthermore, one more switch is eliminated compared with reduced-switch distributed modulator in order to further reduce the loss. The slow-wave structure of DiCAD is also helpful for compactness and loss reduction. Simulation shows this OOK modulator can achieve $3 \mathrm{~dB}$ low loss, $25 \mathrm{~dB}$ $\mathrm{ON} / \mathrm{OFF}$ ratio, which is the best performance compared traditional OOK modulators.

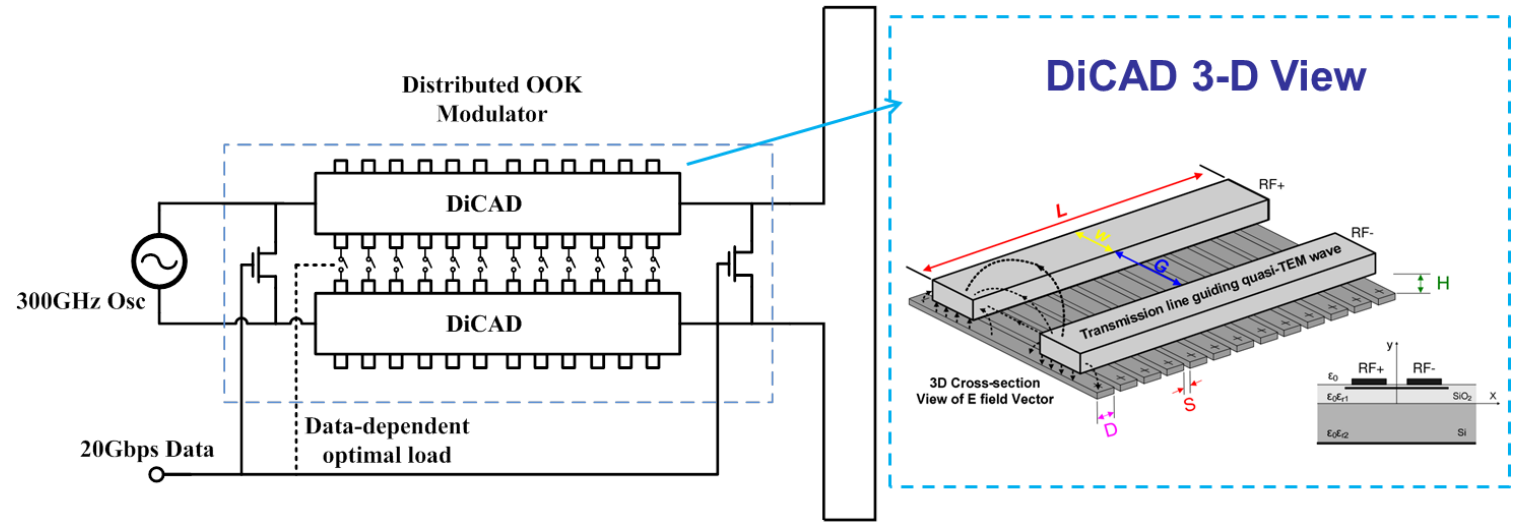

Figure 5. Distributed dynamic optimal load OOK modulator

\section{Conclusion and Risk of Failure}

A new terahertz CMOS OOK modulator with DiCAD is proposed. It is able to achieve low loss, high ON/OFF ratio and high data rate without consuming any DC power. The simulation is done by HFSS and cadence virtuoso in 28nm CMOS process. The proposed modulator can substitute for a PA, which is the most energy-hungry, low efficiency block in a system, and an up-conversion mixer in a transmitter. A much more efficient terahertz OOK transmitter can be implemented. It is anticipated that this will make 20Gbps shortrange wireless data transmission at terahertz regime. The features of different types of OOK modulators are summarized in Table 1 in terms of loss, bandwidth, OFF state isolation and ON/OFF Ratio. It might need several iterations to 


\begin{tabular}{ccccc}
\hline \hline Modulator & Loss & Bandwidth & $\begin{array}{c}\text { OFF state } \\
\text { Isolation }\end{array}$ & $\begin{array}{c}\text { ON/OFF } \\
\text { Ratio }\end{array}$ \\
\hline $\begin{array}{c}\text { Gilbert Based OOK } \\
\text { Modulator }\end{array}$ & High & Low & Low & Low \\
\hline $\begin{array}{c}\text { Distributed-switch } \\
\text { OOK Modulator }\end{array}$ & High & High & High & Moderate \\
\hline $\begin{array}{c}\text { Reduced-switch } \\
\text { Distributed } \\
\text { Modulator }\end{array}$ & Low & High & Moderate & Moderate \\
\hline $\begin{array}{c}\text { Proposed OOK } \\
\text { Modulator with } \\
\text { DiCAD }\end{array}$ & Low & High & High & High \\
\hline \hline
\end{tabular}

Table 1. Features Summary of Different OOK Modulators

The risk of failure mainly comes from the inaccuracy of transistor model at $300 \mathrm{GHz}$ because no one has verified which way is the most accurate and efficient procedure to model the transistor, DiCAD and other passive components behaviors at 300GHz. Several iterations might need to adjust extraction and EM model of transistor, DiCAD and other passive components.

\section{Reference}

[1] A. Tang, H. Wu, and M.-C. Frank Chang, "A 245GHz, 2.6mW/pixel Antenna-less CMOS Imager with $0.7 \mathrm{fW} / \mathrm{Hz}$ NEP and 3.5m Back Backscattered Range", IEEE Asian Solid-State Circuit Conference (A-SSCC 2012), Nov. 12-14, 2012

[2] Woolard, D.; Kaul, R., "Terahertz electronics for chemical and biological warfare agent detection," Microwave Symposium Digest, 1999 IEEE MTT-S International , vol.3, pp.925,928 vol.3, 13-19 June 1999

[3] S. Cherry, Edholm's law of bandwidth, IEEE Spectr. 41, 502004

[4] Kallfass, I., "All Active MMIC-Based Wireless Communication at $220 \mathrm{GHz}$," Terahertz Science and Technology, IEEE Transactions, vol.1, pp.477,487, Nov. 2011

[5] Hong-Yeh Chang; Ming-Fong Lei, "A 46-GHz Direct Wide Modulation Bandwidth ASK Modulator in 0.13- $\mu \mathrm{m}$ CMOS Technology," Microwave and Wireless Components Letters, IEEE , vol.17, no.9, pp.691,693, Sept. 2007

[6] Xu, Z.; Gu, Q.J.; Yi-Cheng Wu; Tang, A.; M.-C.F., "D-band CMOS transmitter and receiver for multi-giga-bit/sec wireless data link," Custom Integrated Circuits Conference (CICC), 2010 IEEE , vol., no., pp.1,4, 19-22 Sept. 2010

[7] Oncu, A.; Takano, K.; Fujishima, M., "8Gbps CMOS ASK modulator for $60 \mathrm{GHz}$ wireless communication," Solid-State Circuits Conference, 2008. A-SSCC '08. IEEE Asian, vol., no., pp.125,128, 3-5 Nov. 2008

[8] LaRocca, T.; Sai-Wang Tam; Daquan Huang; Qun Gu; Socher, E.; Hant, W.; Chang, F., "Millimeter-wave CMOS digital controlled artificial dielectric differential mode transmission lines for reconfigurable ICs," Microwave Symposium Digest, 2008 IEEE MTT-S International , vol., no., pp.181,184, 15-20 June 2008

[9] A. Tang et al., "A 65nm CMOS $140 \mathrm{GHz} 27.3 \mathrm{dBm}$ EIRP transmit array with membrane antenna for highly scalable multi-chip phase arrays," IEEE International Microwave 
Symposium 2014.

[10] A. Tang et al., "A $200 \mathrm{GHz}$ 16-pixel focal plane array imager using CMOS super regenerative receivers with quench synchronization," IEEE International Microwave Symposium 2012.

[11] A. Tang et al., "A $95 \mathrm{GHz}$ centimeter scale precision confined pathway system-onchip navigation processor for autonomous vehicles in $65 \mathrm{~nm}$ CMOS," IEEE International Microwave Symposium 2015.

[12] Tong Zhang et al., "A Simple System for Measuring Antenna Radiation Patterns in the Wi-Fi Band," in IEEE Antennas and Propagation Magazine, vol. 55, no. 1, pp. 191-202, Feb. 2013.

[13] H. Wu et al., "A Current-Mode mm-Wave direct-conversion receiver with $7.5 \mathrm{GHz}$ Bandwidth, $3.8 \mathrm{~dB}$ minimum noise-figure and $+1 \mathrm{dBm} \mathrm{P} 1 \mathrm{~dB}$, out linearity for high data rate communications," 2013 IEEE Radio Frequency Integrated Circuits Symposium (RFIC), Seattle, WA, 2013, pp. 89-92.

[14] Z. Z. Chen et al., "A wide-band 65nm CMOS 28-34 GHz synthesizer module enabling low power heterodyne spectrometers for planetary exploration," 2015 IEEE MTT-S International Microwave Symposium, Phoenix, AZ, 2015, pp. 1-3.

[15] Y. Zhao et al., "A 0.56 THz Phase-Locked Frequency Synthesizer in $65 \mathrm{~nm}$ CMOS Technology," in IEEE Journal of Solid-State Circuits, vol. 51, no. 12, pp. 3005-3019, Dec. 2016.

[16] R. Al Hadi, Y. Zhao, Y. Li, Y. Du, and M.-C. F. Chang, "Retroactive terahertz displacement sensor in a standard 65nm CMOS technology", in Proc. OSA Conf. Lasers and Electro-Optics (CLEO), San Jose, CA, Jun. 2016, pp. 1-3.

[17] R. A. Hadi et al., "A spectral profiling method of mm-wave and terahertz radiation sources," 2016 IEEE MTT-S International Microwave Symposium (IMS), San Francisco, CA, 2016, pp. 1-3.

[18] H. Wu, N. Y. Wang, Y. Du and M. C. F. Chang, "A Blocker-Tolerant Current Mode 60GHz Receiver With 7.5-GHz Bandwidth and 3.8-dB Minimum NF in 65-nm CMOS," in IEEE Transactions on Microwave Theory and Techniques, vol. 63, no. 3, pp. 1053-1062, March 2015.

[19] Y. Li et al., "A multi-band low-noise transmitter with digital carrier leakage suppression and linearity enhancement," in IEEE Transactions on Circuits and Systems I: Regular Papers, vol. 60, no. 5, pp. 1209-1219, May 2013.

[20] Z. Z. Chen et al., "Digital PLL for phase noise cancellation in ring oscillator-based I/Q receivers," 2016 IEEE Symposium on VLSI Circuits (VLSI-Circuits), June 2016.

[21] A. Tang et al., "CMOS (Sub)-mm-Wave System-on-Chip for exploration of deep space and outer planetary systems," Proceedings of the IEEE 2014 Custom Integrated Circuits Conference, San Jose, CA, 2014, pp. 1-4.

[22] Lv Jingjing Du Li(School of Information Science and Engineering of Southeast University,Nanjing 210096, China); Vehicular Collision Avoiding System Based on Two Ultrasonic Receivers[J];Value Engineering;2010-22 
[23] L. Du, Y. Zhang, C. C. Liu, A. Tang, F. Hsiao and M. C. F. Chang, "A 2.3-mW 11-cm Range Bootstrapped and Correlated-Double-Sampling Three-Dimensional Touch Sensing Circuit for Mobile Devices," in IEEE Transactions on Circuits and Systems II: Express Briefs, vol. 64, no. 1, pp. 96-100, Jan. 2017.

[24] L. Du, Y. Zhang, F. Hsiao, A. Tang, Y. Zhao, Y. Li, J. Chen, L. Huang, M.-C. F. Chang, "A 2.3mW 11 cm Range Bootstrapped and Correlated Double Sampling (BCDS) 3D Touch Sensor for Mobile Devices," IEEE International Solid-State Circuits Conference, pp. 122123, Feb. 22-26, 2015.

[25] L. Du et al., "Invited - Airtouch: A novel single layer 3D touch sensing system for human/mobile devices interactions," 2016 53nd ACM/EDAC/IEEE Design Automation Conference (DAC), Austin, TX, 2016.

[26] W. H. Cho et al., "A 5.4-mW 4-Gb/s 5-band QPSK transceiver for frequency-division multiplexing memory interface," 2015 IEEE Custom Integrated Circuits Conference (CICC), San Jose, CA, 2015, pp. 1-4.

[27] W. H. Cho et al., "A 38mW 40Gb/s 4-lane tri-band PAM-4 / 16-QAM transceiver in 28nm CMOS for high-speed Memory interface," 2016 IEEE International Solid-State Circuits Conference (ISSCC), San Francisco, CA, 2016, pp. 184-185.

[28] Y. Du et al., "A $16 \mathrm{~Gb} / \mathrm{s} 14.7 \mathrm{~mW}$ tri-band cognitive serial link transmitter with forwarded clock to enable PAM-16 / 256-QAM and channel response detection in $28 \mathrm{~nm}$ CMOS," 2016 IEEE Symposium on VLSI Circuits (VLSI-Circuits), Honolulu, HI, 2016, pp. 1-2.

[29] Y. Du et al., "A 16-Gb/s 14.7-mW Tri-Band Cognitive Serial Link Transmitter With Forwarded Clock to Enable PAM-16/256-QAM and Channel Response Detection," in IEEE Journal of Solid-State Circuits , vol.PP, no.99, pp.1-12.

[30] B. Hu et al., "A Capacitor-DAC-Based Technique For Pre-Emphasis-Enabled MultiLevel Transmitters," in IEEE Transactions on Circuits and Systems II: Express Briefs, vol.PP, no.99, pp.1-1.

[31] Du, Yuan. (2016). Cognitive Serial Interface with Multi-Band Signaling and Channel Learning Mechanism. UCLA: Electrical Engineering 0303. Retrieved from: http://escholarship.org/uc/item/8vs373c5

[32] Y. Li et al., "Transconductance enhancement method for operational transconductance amplifiers," Electronics Letters, vol. 46, no. 19, pp1321-1323, 2010.

[33] Y. Li et al., "Analysis and implementation of an improved recycling folded cascode amplifier," Journal of Semiconductors, vol. 33, no. 2, 2012.

[34] Y. Li et al., "A subthreshold MOSFET bandgap reference with ultra-low power supply voltage," IEEE 9th International Conference on ASIC (ASICON), Shanghai, 2011.

[35] Y. Li et al., "A $0.6 \mathrm{ppm} / \mathrm{C}$ current-mode bandgap with second-order temperature compensation," IEEE 9th International Conference on ASIC (ASICON), Shanghai, 2011. [36] Y. Li et al., "Carrier synchronisation for multiband RF interconnect (MRFI) to facilitate chip-to-chip wireline communication," in Electronics Letters, vol. 52, no. 7, pp. 535-537, 2016. 
